\title{
Optically Selected GRB Afterglows
}

\author{
F. Malacrino* and J-L. Atteia* \\ * Laboratoire d'Astrophysique Observatoire Midi-Pyrénées, 14 avenue Edouard Belin, 31400 \\ Toulouse, France
}

\begin{abstract}
Since November 2004, we attempt to detect GRB optical afterglows in near real-time on images taken at the Canada France Hawaii Telescope within the Very Wide Survey, component of the CFHT Legacy survey. To do so, a Real Time Analysis System automatically and quickly analyzes MegaCAM images and extracts from them a list of photometrically and astrometrically variable objects which is then validated by a member of the collaboration. Each month, we repeatedly observe 15 to 30 square degrees down to magnitude i' $=22.5$. A few objects are classified as candidates and analyzed more deeply, and statistics are done showing the treatment's performance.
\end{abstract}

Keywords: gamma-ray bursts, observation and data reduction techniques, image processing PACS: $98.70 . \mathrm{Rz}, 95.75 .-\mathrm{z}$, 95.75.Mn

\section{THE SURVEY}

The Canada France Hawaii Telescope is a $3.6 \mathrm{~m}$ telescope located on the Mauna Kea in Hawaii. Built in the late 70's, it has been recently equipped with a new instrument, Megacam, a full square degree CCD imager.

The Very Wide Survey covers 1200 square degrees down to i' $=22.5$, through 3 filters (r', g', i'). Initially conceived to discover and follow 2000 Kuiper Belt Objects, its observing strategy (Fig 1) is well suited to detect optical afterglows.

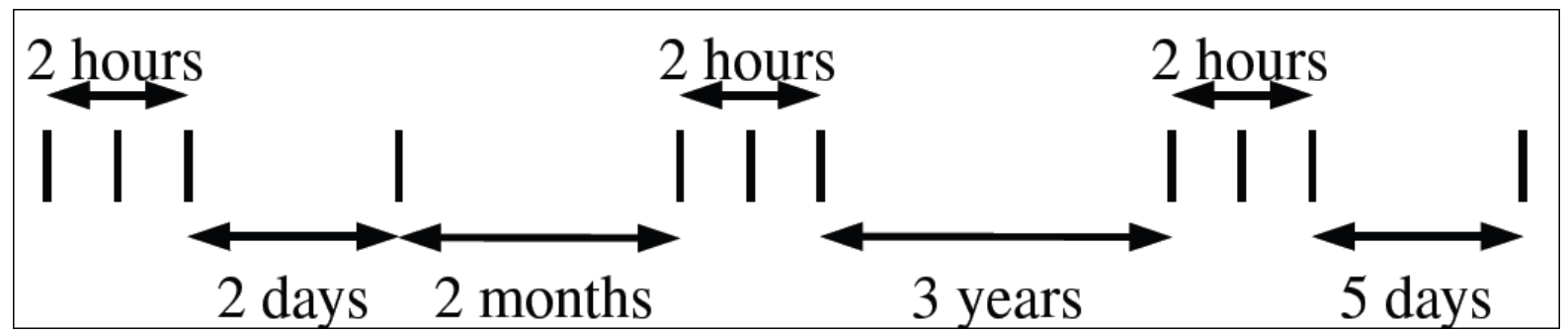

FIGURE 1. Observational strategy for one field (each vertical line is for one exposure, exposure time depends on the filter, but is typically of the order of one hundred seconds)

Each field is observed several times. This recurrence can be used to compare images between them in order to detect variable or transient objects.

${ }^{1}$ on behalf of the GRB RTAS collaboration 


\section{THE REAL TIME ANALYSIS}

\section{Catalog Process}

This part of the process consists of reducing the useful information from 700 Mo to a few ten Mo and to prepare all the useful data for the comparison process. The pipeline automatically checks the presence of a new image as soon as it has been pre-processed by Elixir and starts the treatment for each of the 36 CCDs.

It consists of converting FITS images to JPEG format, extracting FITS headers and creating a catalog of objects, containing their main characteristics. These objects are then sorted according to their astrophysical properties (stars, galaxies, cosmic rays, etc..) and astrometically matched with the USNO catalog. Results are finally summarized on a web page to be checked and validated by a collaboration member. Images treated in this way can then be used in the comparison process.

\section{Comparison Process}

The goal of this process is first to list all the possible comparisons between images of the same field. To be possible, a comparison must involve images with the same filter and exposure time. In a second step, the process checks if the field has already been observed during this run, and in this case starts the comparison between the two best quality images of each night.

The comparison process classifies objects into two categories: matched and single objects. Matched objects are used for photometric inter-calibration. Asteroids are detected among single objects. At last, a search of matched objects having significant variation in luminosity is done.

All the information about variable objects and asteroids is gathered on a interactive web page. Then a member of the collaboration has to reject false detections or to validate objects as really variable. This validation process typically takes 2-3 hours for 1 run.

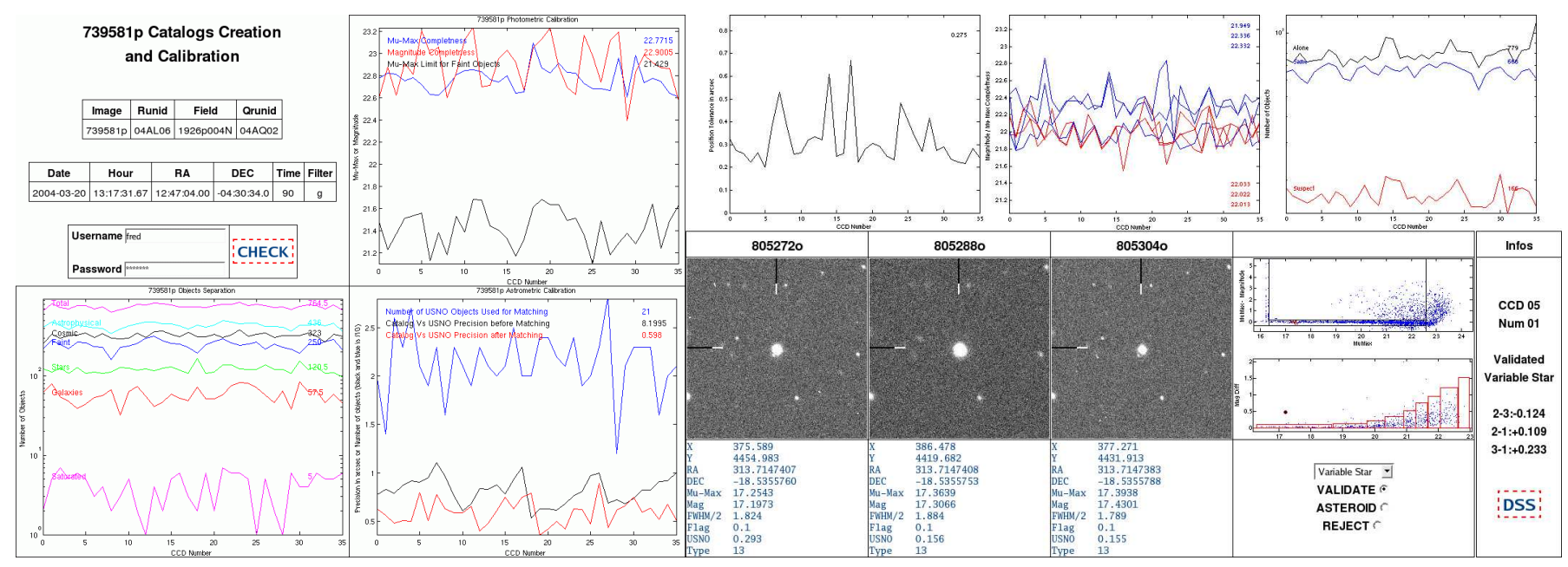

FIGURE 2. Example of a "catalog creation" web page (left) and a "triple comparison" web page (right) 


\section{THE POST ANALYSIS}

At the end of each run, statistics are performed on catalogs, comparisons and validation. These statistics allow a more comprehensive view of the treatment and the possibility of comparing different runs. Ultimately the performances of the survey will be evaluated on a population of simulated GRB afterglows to constrain the rate of GRB afterglows.

Catalogs. Table 1 shows statistics for the catalog process of the MegaCAM run 05BQ07. The Very Wide survey usually works by sets of 16 or more fields, that's why images are classified by sets. We can notice that i' filter images contain twice more objects than r' filter ones, although the completness magnitudes are almost the same. It can also be noted that the last $i$ ' filter set is worse compared with the first two due to undefringe images.

TABLE 1. Statistics for 05BQ07 catalogs

\begin{tabular}{cccccccc}
\hline Filter & Set & Date & $\begin{array}{c}\text { Number of } \\
\text { Images }\end{array}$ & $\begin{array}{c}\text { Square } \\
\text { Degrees }\end{array}$ & $\begin{array}{c}\text { USNO } \\
\text { Precision }\end{array}$ & $\begin{array}{c}\text { Completness } \\
\text { Magnitude }\end{array}$ & $\begin{array}{c}\text { Objects per } \\
\text { Square Degrees }\end{array}$ \\
\hline r' & 1 & $2005-10-25$ & 18 & 16.018 & 0.60 & 22.53 & 15652 \\
r' & 2 & $2005-10-25$ & 16 & 14.288 & 0.59 & 22.40 & 14415 \\
r' & 3 & $2005-10-25$ & 17 & 15.165 & 0.60 & 22.34 & 14139 \\
r' & 4 & $2005-10-26$ & 17 & 15.266 & 0.60 & 22.19 & 12613 \\
i' & 1 & $2005-10-26$ & 16 & 14.438 & 0.62 & 22.32 & 28926 \\
i' & 2 & $2005-10-26$ & 16 & 14.438 & 0.62 & 22.39 & 30221 \\
i' & 3 & $2005-10-26$ & 17 & 15.341 & 0.62 & 22.10 & 28499 \\
\hline
\end{tabular}

Comparisons. Using these catalogs, 32 triple comparisons have been processed, 16 for each filter. Triple comparisons involves images of the same night. r' filter triple comparisons are very impressive, with only $0.05 \%$ of photometrically variable objects and many asteroids detected down to magnitude r' $=22.1$. Unfortunately, the bad third image of the i' filter set leads to a huge number of photometrically variable objects, and this set of triple comparisons hasn't been validated by the team due to many false detections.

\begin{tabular}{|c|c|c|c|c|c|c|c|c|c|c|c|c|}
\hline \multicolumn{13}{|c|}{ Triple Comparisons } \\
\hline $\begin{array}{l}\text { Exposure } \\
\text { Time }\end{array}$ & Filter & Set & Date & Hour & $\begin{array}{c}\text { Number of } \\
\text { Comparisons }\end{array}$ & $\begin{array}{l}\text { Square } \\
\text { Degrees }\end{array}$ & $\begin{array}{l}\text { Position } \\
\text { Tolerance }\end{array}$ & $\begin{array}{c}\text { Limit } \\
\text { Magnitude }\end{array}$ & $\begin{array}{l}\text { Objects } \\
\text { per }^{\circ 2}\end{array}$ & $\begin{array}{c}\text { Dif } \\
\text { Objects }\end{array}$ & Asteroids & $\begin{array}{c}\text { Dif Objects } \\
\text { for } 10000 \\
\text { objects per }^{\circ 2}\end{array}$ \\
\hline 110 & $\mathrm{r}$ & 1 & 2005-10-25 & $11: 36: 09.37$ & 16 & 14.037 & 0.27 & 22.07 & 13186 & 93 & 736 & 5.02 \\
\hline 180 & $\mathrm{i}$ & 2 & $2005-10-26$ & $5: 59: 28.08$ & 16 & 14.438 & 0.36 & 21.81 & 26010 & 814 & 446 & 21.68 \\
\hline \multicolumn{13}{|c|}{ Double Comparisons } \\
\hline $\begin{array}{l}\text { Exposure } \\
\text { Time }\end{array}$ & Filter & Set & Date 1 & Date 2 & $\begin{array}{c}\text { Number of } \\
\text { Comparisons }\end{array}$ & $\begin{array}{c}\text { Square } \\
\text { Degrees }\end{array}$ & $\begin{array}{l}\text { Position } \\
\text { Tolerance }\end{array}$ & $\begin{array}{c}\text { Limit } \\
\text { Magnitude }\end{array}$ & $\begin{array}{l}\text { Objects } \\
\text { per }^{\circ 2}\end{array}$ & $\begin{array}{c}\text { Dif } \\
\text { Objects }\end{array}$ & $\begin{array}{l}\text { Alone } \\
\text { Objects }\end{array}$ & $\begin{array}{c}\text { Dif Objects } \\
\text { for } 10000 \\
\text { objects per } \\
{ }^{\circ} 2\end{array}$ \\
\hline 110 & $\mathrm{r}$ & 1 & 2005-10-26 & $X X X X-X X-X X$ & 1 & 0.902 & 0.35 & 21.99 & 10228 & 5 & 0 & 5.42 \\
\hline 110 & $\mathrm{r}$ & 2 & $2005-10-26$ & $2005-10-25$ & 16 & 14.213 & 0.33 & 22.05 & 12289 & 91 & 11 & 5.21 \\
\hline
\end{tabular}

FIGURE 3. Statistics for 05BQ07 comparisons 
Double comparison statistics only concern r' filter images. The first set is insignificant, as it contains only one double comparison of 2 images of the same night. On the contrary, the second set looks like what we get in case of perfect double comparisons. Once again, the process selects approximatively $0.05 \%$ of luminosity variable objects down to magnitude $\mathrm{r}^{\prime}=22$, and 11 objects which were classified as matched in the corresponding triple comparison, and are not present in the image taken one day later. The behaviour of these objects looks like the expected behaviour of GRB afterglows, showing that we can indeed detect GRB afterglows.

Validation. In the last part, we combine triple and double comparisons, and their corresponding validations, in order to compute statistics for asteroids and variable objects per field, as we consider that fields observed 2 days apart are not independent.

\begin{tabular}{|c|c|c|c|c|c|c|c|c|c|c|c|c|c|c|c|c|}
\hline \multicolumn{17}{|c|}{ Variable Objects } \\
\hline $\begin{array}{c}\text { Exposure } \\
\text { Time }\end{array}$ & Filter & Nature & \begin{tabular}{|c} 
Total \\
Number
\end{tabular} & Rejected & Validated & Cosmic & \begin{tabular}{|c|} 
CCD \\
Defect
\end{tabular} & $\begin{array}{l}\text { CCD } \\
\text { Edge }\end{array}$ & Seeing & Contaminated & Faint & Other & Galaxy & $\begin{array}{c}\text { Variable } \\
\text { Star }\end{array}$ & TNO & Candidate \\
\hline 110 & $r$ & Triple+Double & 181 & 160 & 21 & 15 & 19 & 7 & 98 & 20 & 0 & 2 & 1 & 17 & 0 & 2 \\
\hline
\end{tabular}

FIGURE 4. Statistics for 05BQ07 variable objects validation

Only $12 \%$ of objects classified as variable by the process are really variable, and most of them are variable stars. More than $50 \%$ of the detections are due to seeing variations, despite corrections applied by the process. 5 interesting candidates have been identified which were all discarded by comparison with previous images of the field.

\section{CONCLUSION}

We have shown that optical afterglow detection is possible using Megacam images from the Very Wide survey at the CFHT and a real time comparison pipeline. About 1 to 10 afterglows per year can be reasonably expected. The real time process has started one year ago, and 12 runs have been succesfully processed, but unfortunately without any strong afterglow candidate. Now, we have started the complete reprocessing of the whole Very Wide survey runs since the beginning of the legacy survey in April 2003. This study will allow us to compute a huge database of objects which will be very useful to plot luminosity function of the most interesting objects, and to strongly constrain the rate of orphan GRB afterglows and their beaming factor. Our work is continuing with new images taken every month, and we recently submitted a proposal for the next semester, which is specifically dedicated to the search of orphan GRB afterglows and will have a efficiency 2-3 times greater than the Very Wide Survey for this task.

Please visit http://www. cfht.hawaii.edu/ grb for more informations on this research.

\section{ACKNOWLEDGMENTS}

The authors gratefully acknowledge the support of the CFHT staff for the steady operation of the RTAS. 